\title{
Bacteriemia por Staphylococcus aureus adquirida en la comunidad: comportamiento clínico y severidad en niños
}

\section{Community-acquired Staphylococcus aureus bacteriology: clinical behavior and severity in children}

\author{
Soraya Araya ${ }^{1,2}$, Avelina Troche ${ }^{2}$, Rocio Benitez ${ }^{2}$, Sara Amarilla ${ }^{2}$, Gabriela Sanabria ${ }^{2}$, Limpia \\ Ojeda $^{2}$, Sandra Cubas $^{2}$, Claudia Zarate ${ }^{2}$, Juan Irala ${ }^{2}$, Silvio Apodaca ${ }^{2}$ y Antonio Arbo ${ }^{2,3}$
}

\section{RESUMEN}

Introducción: La bacteriemia por Staphylococcus aureus (SA) constituye una de las más graves infecciones de la edad pediátrica. Objetivos: Evaluar las características epidemiológicas, clínicas y laboratoriales de niños con bacteriemias por SA adquiridas en la comunidad (SAAC), identificar factores de riesgos asociados a mortalidad. Materiales y métodos: Estudio retrospectivo en el que se incluyeron pacientes (pts) de $\leq 16$ años, hospitalizados entre 2010-2018, con dx de bacteriemias por SAAC. Los datos clínicos y laboratoriales fueron introducidos en una base de datos de Excel y formateados para análisis. Resultados: Se identificaron 117 pts con una edad media de 56+53 meses, de los cuales 81 fueron $\leq 5$ años. La bacteriemia se asoció principalmente a neumonía ( $47 \%$ de los casos), presentando choque el 38\% e ingreso a UCI el $40 \%$ de los pts. En el 27\% (32/117) la bacteriemia fue por SAMR. La edad $<5$ años $(\mathrm{p}=0.0001)$, presencia de choque $(\mathrm{p}=0001)$, hospitalización en UCI ( $\mathrm{p}=0.002$, OR 3.58, IC95\% 1.5-8.3) y la mortalidad ( $\mathrm{p}=0.03$, OR 2.65, IC95\% 1.05-6.7) se asociaron a infección por SAMR. La mortalidad de esta serie fue del $21 \%$ (25/117). La presencia de comorbilidad ( $p=0.006$, OR3.66, IC95\% 1.4-92), choque ( $\mathrm{p}=0.0001$, OR 87.6, IC 95\% 11.5-687.7), focos múltiples infecciosos ( $\mathrm{p}=0.007, \mathrm{OR} 3.46$, IC $95 \% 1.3-8.9)$, aislamiento de SAMR ( $p=0.03$, OR 2.65 ,IC $95 \% 1.1-6.7)$, y trombocitopenia $<100000 / \mathrm{mm}^{3}(\mathrm{p}=0.0001$, OR 25.3, IC 95\% 5-128) se asociaron a mortalidad. Conclusiones: El presente estudio muestra la severidad de la bacteriemia por SA. La resistencia a meticilina, la comorbilidad, la presencia de focos múltiples y choque se identificaron como factores asociados a mortalidad.

Palabras clave: Bacteriemia, Staphylococcus aureus, niños.

\section{ABSTRACT}

Introduction: Bacteremia due to Staphylococcus aureus (SA) is one of the most serious infections in the pediatric population. Objectives: To evaluate the epidemiological, clinical and laboratory characteristics of children with bacteremia due to SA acquired in the community (SAAC) and to identify risk factors associated with mortality. Materials and methods: This was a retrospective study in which patients (pts) $\leq 16$ years, hospitalized between 20102018, with $\mathrm{dx}$ of bacteremia by SAAC were included. Clinical and laboratory data were entered into an Excel database and formatted for analysis. Results: We identified 117 pts with an average age of $56 \pm 53$ months, of which 81 were $\leq 5$ years. Bacteremia was associated mainly with pneumonia ( $47 \%$ of the cases), presenting with shock in $38 \%$ and admission to the ICU in $40 \%$ of the pts. In $27 \%$ $(32 / 117)$ the bacteremia was caused by MRSA. Age $<5$ years $(p=0.0001)$, presence of shock $(p=0001)$, hospitalization in the ICU ( $\mathrm{p}=0.002$, OR $3.58,95 \%$ CI 1.5 8.3 ) and mortality ( $p=0.03$, OR $2.65,95 \%$ CI 1.05-6.7) were associated with MRSA infection. The mortality in this series was $21 \%(25 / 117)$. The presence of comorbidities $(\mathrm{p}=$ 0.006, OR3.66, 95\% CI 1.4-92), shock ( $\mathrm{p}=0.0001$, OR 87.6, $95 \%$ CI 11.5-687.7), multiple infectious foci $(\mathrm{p}=0.007$, OR3.46, 95\% CI 1.3-8.9), MRSA isolation ( $p=0.03$, OR 2.65, $95 \%$ CI 1.1-6.7), and thrombocytopenia $<100000 / \mathrm{mm} 3$ ( $\mathrm{p}=$ 0.0001, OR 25.3, 95\% CI 5-128) were associated with mortality. Conclusions: This study shows the severity of SA bacteremia. Methicillin resistance, comorbidities, the presence of multiple infectious foci and shock were identified as factors associated with mortality.

Keywords: Bacteremia, Staphylococcus aureus, children.

\footnotetext{
${ }^{1}$ Universidad Católica de Asunción. Lambaré, Paraguay.

${ }^{2}$ Instituto de Medicina Tropical. Asunción, Paraguay.

${ }^{3}$ Universidad Nacional de Asunción, San Lorenzo, Paraguay.

Correspondencia: Soraya Araya Correo: sorayarayagmail.com

Conflicto de interés: Los autores declaran no poseer conflicto de interés

Recibido: 26/10/2018 Aceptado: 29/12/2018

DOI: https://doi.org/10.31698/ped.45032018002
} 


\section{INTRODUCCIÓN}

El Staphylococcus aureus representa actualmente uno de los más importantes patógenos de la edad pediátrica, y es responsable de una amplia variedad de cuadros clínicos que van desde infecciones superficiales a cuadros graves como la endocarditis y el choque séptico ${ }^{(1)}$.

La Incidencia de Bacteriemia por Staphylococcus aureus en niños varía de 6 a 20 por 100 000, con una tasa de hospitalización de 1,5 a 3,5 por 1000 ingresos. En el Paraguay en un estudio reciente sobre bacteriemias de origen comunitario el cual incluyó 187 niños con bacteriemia, las bacteriemias por Gram Positivos fueron más frecuentes que las bacteriemias por Gram Negativos $(91,4 \%$ vs $8,6 \%$, $\mathrm{p}<0,05)$, constituyendo el S.aureus el $34 \%$ de los aislamientos ${ }^{(2)}$.

En la última década se ha observado a nivel mundial la emergencia de infecciones comunitarias causadas por cepas de Staphylococcus aureus con resistencia a las isoxazolpenicilinas, conocidas como S. aureus meticilino-resistentes (SAMR). Estas cepas muestran un patrón de sensibilidad a los antibióticos diferente de las de origen hospitalario, presentando resistencia a todas las isoxazolpenicilinas (meticilina, oxacilina, dicloxacilina, nafcilina, flucloxacilina), sensibilidad variable a la clindamicina y alta sensibilidad a trimetroprima-sulfametoxazol.

En Estados Unidos, el SAMR, tradicionalmente un patógeno de adquisición en hospitales, se convirtió en una causa cada vez más común de las infecciones de niños y adultos originadas en la comunidad, desde el inicio de la década de los 90, y su prevalencia actual en varias partes de EEUU supera al 60\%. En América Latina los primeros reportes de casos de infecciones adquiridas por SAMR (SAMRAC) tuvieron lugar en Uruguay en el año 2001, tras lo cual los diferentes países del continente, incluyendo el Paraguay, reportaron la presencia de SARM-AC, con prevalencias de resistencia a meticilina del $25 \%$ al $70 \%$ de los aislamientos ${ }^{(3)}$.

Teniendo en cuenta que la gravedad y mortalidad asociada a las infecciones causadas por $S$. aureus adquiridas en la comunidad poseen especial importancia en este nuevo escenario de susceptibilidad antimicrobiana del S. aureus; nos avocamos a analizar las características clínicas actuales en pacientes pediátricos conbacteriemia de origen comunitario por el citado germen

\section{Objetivos}

Evaluar las características epidemiológicas, clínicas y laboratoriales de niños con bacteriemias por $S$. aureus adquiridas en la comunidad (BSAC).

Identificar los factores de riesgos asociados a mortalidad.

\section{MATERIALES Y MÉTODOS}

Tipo de Estudio: Estudio retrospectivo de todas las bacteriemias por $S$. aureus de origen comunitario, diagnosticadas en el Instituto de Medicina Tropical del Paraguay, durante el periodo comprendido de enero de 2010 a enero de 2018.

Criterios de Inclusión: Se incluyeron a los pacientes de 29 días de vida a 16 años, con diagnóstico de egreso de bacteriemias por SAAC.

Criterios de exclusión: Bacteriemias de origen comunitario por otra causa (Streptococcus pneumoniae, Neisseria meningitidis, Haemophilus influenzae u otro germen), pacientes en edad neonataly edad mayor a 16 años.

Bacteriemia por S. aureus adquirida en la Comunidad (BSAC): Se definió por la presencia de un hemocultivo positivo para $S$. aureus y signos compatibles con infección, o la detección de dos muestras positivas de hemocultivos, obtenidos durante las primeras $72 \mathrm{hs}$ de internación del niño. La endocarditis fue definida según los criterios de Duke.

Evolución: Se categorizaron a los pacientes en dos grandes grupos según el desenlace final (vivos o muertos). El óbito fue atribuido a la infección cuando el niño presentaba signos persistentes de infección sistémica refractarios al tratamiento, con hemocultivo positivo para S.aureus. Se analizaron 
las variables clínicas y laboratoriales presentes al ingreso del paciente y se correlacionaron con el desenlace final (vivos o muertos).

Microbiología: Las cepas S. aureus se identificaron mediante las pruebas microbiológicas habituales Sistema Automatizado Vitek $2 C \circledR$. La sensibilidad a meticilina se determinó mediante determinación de la Concentración InhibitoriaMínima (CIM) a Oxacilina por Sistema Vitek $2 C \circledR$, siendo reportadas como resistentes aquellas cepas con CIM iguales o mayores a $4 u \mathrm{~g} / \mathrm{mL}$ y sensibles aquellas menores o iguales a $2 u \mathrm{~g} / \mathrm{mL}$. La detección de la presencia del Gen MLSBinducible (Test "D") también fue determinada por el mismo sistema automatizado.

Análisis Estadístico: Los datos (edad, días de internación) se expresaron como media aritmética. Un valor $p$ menor de 0,05 se consideró significativo. Todos los datos se almacenaron en un programa informativo Epi Info. Los valores fueron expresados en base al total de datos disponibles. La significación estadística se calculó mediante la prueba de X2 o la prueba exacta de Fisher para las variables nominales y la pueba de $t$ de Student para las variables numéricas. El análisis de los factores de riesgos se efectuó por la relación de momios (OR) con Índice de Confianza del $95 \%$.

Ética: Se solicitó autorización al Comité de Ética de la Institución, se respetó la confidencialidad de los pacientes.

\section{RESULTADOS}

Se identificaron 117 bacteriemias por Staphylococcus aureus en niños. La edad media fue $56+53$ meses, siendo $\leq 5$ años $69 \%$ (81/117) y mayores de 5 años $31 \%$ (36/117). Los diagnósticos de ingreso fueron: neumonía, infección de piel y partes blandas, infección osteoarticular, endocarditis, infección de sistema nervioso central y foco múltiple en $47 \%$, $15 \%, 10 \%, 2 \%, 2 \%$ y $24 \%$ respectivamente (Tabla 1 ).

Al analizar la sensibilidad antibiótica de las cepas de SAAC, se encontró que el $27 \%$ fueron resistentes a meticilina (32/117), constatándose resistencia inducible a la Clindamicina (Test " $\mathrm{D}$ " positivo) en el $19 \%(22 / 117)$ de los pacientes. Ninguna cepa fue resistente a vancomicina (Tabla 1).

En cuanto a la gravedad de los niños, presentó choque $38 \%$ (44/117) de los mismos, requiriendo ingreso a Unidad de Cuidados Intensivos el $40 \%$ (47/117) de los casos. La mortalidad de la serie fue 21 $\%$ (25/117), los cuales se produjeron a los $7+6$ días del ingreso (Tabla 1 ).

Tabla 1. Características Clínicas y Laboratoriales de los Niños con BSAC, asociadas a Mortalidad. $\mathrm{N}=117$.

\begin{tabular}{lcccccc}
\hline Variables & $\begin{array}{c}\text { Total } \\
\mathbf{N = 1 1 7}\end{array}$ & $\begin{array}{c}\text { Óbitos } \\
\mathbf{n = 2 5}\end{array}$ & $\begin{array}{c}\text { Vivos } \\
\mathbf{n = 9 2}\end{array}$ & $\mathbf{p}$ & OR & IC 95\% \\
\hline Edad & & & & & & \\
Menor de 1 año & 32 & 10 & 22 & 0.88 & 0.92 & $0.3-2.4$ \\
1 a 5 años & 49 & 7 & 42 & & & \\
6 a 12 años & 22 & 4 & 18 & & & \\
Mayor de 12 años & 14 & 4 & 10 & & & \\
Variables clínicas & & & & & & \\
Comorbilidad & 34 & 13 & 21 & 0.006 & 3.66 & $1.4-9.2$ \\
Choque & 44 & 24 & 20 & 0.000 & 87.6 & $11.5-687.7$ \\
UCl & 47 & 25 & 19 & 0.000 & 0.00 & - \\
Diagnóstico de Ingreso & & & & & & \\
Neumonía & 55 & 11 & 44 & 0.73 & 0.85 & $0.3-2$ \\
IOAR & 13 & 1 & 12 & 0.29 & 0.22 & $0.03-2.2$ \\
Endocarditis & 2 & 1 & 1 & 0.38 & 3.79 & $0.2-62.2$ \\
IPPB & 17 & 0 & 17 & 0.02 & 0.00 & - \\
ISNC & 2 & 1 & 1 & 0.38 & 3.79 & $0.2-62.8$ \\
Foco Múltiple & 28 & 11 & 17 & 0.007 & 3.46 & $1.3-8.9$ \\
Resistencia antibiótica & & & & & & \\
MR & 32 & 11 & 21 & 0.03 & 2.65 & $1.1-6.7$ \\
Clindamicina R & 22 & 7 & 15 & 0.18 & 1.99 & $0.7-5.6$ \\
Laboratorio & & & & & & \\
Saturación O2 < 94 & 27 & 16 & 11 & 0.000 & 13.1 & $4.6-36.7$ \\
GB $>$ 15000 & 42 & 8 & 34 & 0.64 & 0.8 & $0.3-2$ \\
GB < 5000 & 32 & 8 & 24 & 0.55 & 1.33 & $0.5-3.4$ \\
Plaquetas <100000 & 11 & 9 & 2 & 0.000 & 25.3 & $5-128$ \\
Hemoglobina <7 & 9 & 5 & 4 & 0.02 & 5.5 & $1.3-22.3$ \\
\hline
\end{tabular}

$\mathrm{Al}$ establecer la comparación entre pacientes con bacteriemias por SAMR y SAMS, las variables que se asociaron a infección por SAMR fueron laedad menor de 5 años $(\mathrm{p}=0.0001)$, la resistencia a la clindamicina ( $\mathrm{p}=0.001$, OR 4.5,IC95\%1.7-11.9), la presencia de choque $(\mathrm{p}=0001)$, el ingreso a $\mathrm{UCI}(\mathrm{p}=0.002$, OR 3.58, IC95\% 1.5-8.3) y la mortalidad( $\mathrm{p}=0.03$, OR 2.65, IC95\% 1.05-6.7)(Tabla 2).

Tabla 2. Comparación de los pacientes con BSAC por SAMR y SAMS. N=117.

\begin{tabular}{lcccccc}
\hline Variables & $\begin{array}{c}\text { Total } \\
\mathbf{N = \mathbf { 1 1 7 }}\end{array}$ & $\begin{array}{c}\text { SAMR } \\
\mathbf{n = 3 2}\end{array}$ & $\begin{array}{c}\text { SAMS } \\
\mathbf{n = 8 5}\end{array}$ & $\mathbf{p}$ & OR & IC 95\% \\
\hline Edad $<5$ & 19 & 18 & 1 & $0.000 *$ & 108 & $13.3-874.6$ \\
IOA & 13 & 5 & 8 & 0.33 & 1.78 & $0.5-5.9$ \\
Endocarditis & 2 & 1 & 1 & 0.47 & 2.7 & $0.1-44.6$ \\
IPPB & 17 & 10 & 7 & 0.001 & 5.06 & $1.7-14.8$ \\
ISNC & 2 & 1 & 1 & 0.47 & 2.7 & $0.1-44.6$ \\
Foco Múltiple & 28 & 10 & 18 & 0.25 & 1.69 & $0.6-4.2$ \\
\hline Clindamicina R & 22 & 12 & 10 & 0.001 & 4.5 & $1.7-11.9$ \\
UCI & 47 & 20 & 27 & 0.002 & 3.58 & $1.5-8.3$ \\
Choque & 44 & 32 & 12 & 0.000 & - & - \\
Mortalidad & 25 & 11 & 14 & 0.03 & 2.65 & $1.05-6.7$ \\
\hline
\end{tabular}


Uno de los objetivos del estudio fue analizar los factores asociados a mortalidad de la bacteriemia por S. aureus. Al comparar los pacientes que sobrevivieron con los que fallecieron, los factores asociados a mortalidad fueron la comorbilidad ( $\mathrm{p}=0.006$, OR3.66, IC95\% 1.4-92), choque( $\mathrm{p}=0.0001$, OR 87.6, IC 95\% 11.5$687.7)$ infección a múltiple foco $(\mathrm{p}=0.007, \mathrm{OR} 3.46$, IC $95 \%$ 1.3-8.9) infección por SAMR ( $\mathrm{p}=0.03$, OR 2.65,IC $95 \% 1.1-6.7)$, Saturación de O2 $<94 \%(\mathrm{p}=0.0001$, OR 13.1, IC95\% 4.6-36,7), plaquetas $<100000$ ( $\mathrm{p}=0.0001$, OR 25.3, IC 95\% 5-128) (Tabla 2).

\section{DISCUSIÓN}

Las bacteriemias de origen comunitario por S. aureus poseen gran relevancia clínica en la actualidad, sobre todo en aquellos países que cuentan con esquemas de vacunación contra el $H$. influenzae y el Streptococcus pneumoniae ${ }^{(3)}$.

Actualmente el $S$. aureus representa un patógeno prevalente entre las causas de bacteriemia en niños en nuestro país. En un estudio reciente realizado en Paraguay que incluyó a 187 niños con bacteriemia de origen comunitario, el S.aureus representó el $34 \%$ de los aislamientos ${ }^{(4)}$. Otros países también reportan aumento de la frecuencia de $S$. aureus en bacteriemias. En ese sentido, en Estados Unidos el S. aureus representó el $12 \%$ de los aislamientos ${ }^{(5)}$, mientras que en Israel el S. aureus ocupó el tercer lugar en frecuencia, situación observada también en Dinamarca y Australia $^{(6,7)}$. En países desarrollados como Dinamarca o Finlandia la incidencia del S.aureus fue de 8,4 casos y 5 casos por 100000 niños, respectivamente ${ }^{(6)}$. Existen pocos estudios latinoamericanos que hayan analizado la bacteriemia por $S$. aureus en niños. Reporte de una cohorte de niños de Argentina ha informado una incidencia de 1 caso por cada 1000ingresos hospitalarios, similar a lo observado en nuestro país ${ }^{(7,8)}$.

En la presente serie de bacteriemias por S. aureus, la presentación clínica más frecuente observada al ingresofue la neumonía, otras localizaciones fueron la infección de piel y partes blandas que se observó en el 15\% de los casos, la osteoarticular en el $11 \%$, constatándose la afección de múltiples focos en el $25 \%$ de los casos, siendo infrecuente la endocarditis $(1,7 \%)$. En otras series de bacteriemias por $S$. aureus de países desarrollados la presentación clínica más frecuente fue la infección osteoarticular (31\%), seguida por la infección de piel y partes blandas $(16 \%)$ e infección de foco múltiple $(14 \%)$, siendo también infrecuente la endocarditis (2\%); llama la atención que en pacientes adultos con bacteriemia por S. aureus la endocarditis fue frecuente (30\%) lo que demuestra el comportamiento diferente del $S$. aureus en niños ${ }^{(10,11)}$.

El conocimiento del perfil de sensibilidad antibiótica del $S$. aureus tiene una enorme trascendencia a la hora de optar por la mejor alternativa terapéutica, en la que la decisión adecuada impacta profundamente en la reducción de la mortalidad de los pacientes críticos ${ }^{(9)}$ cuando el antibiótico instaurado en la primera hora de internación, resulta concordante con la sensibilidad antibiótica del agente causal. En la presente serie se constató meticilino resistencia en el $27 \%$ de las bacteriemias y resistencia inducible en el $19 \%$ a la clindamicina.

Si bien en nuestra serie los pacientes en edad neonatal fueron excluidos, el $69 \%$ de los niños fueron menores de 5 años de edad. Otras series latinoamericanas reportaron mayor frecuencia de BSAC en niños menores de 1 año ${ }^{(8)}$.

La gran mayoría de los estudios queanalizaron la mortalidad en Bacteriemias por S.aureus se llevó a cabo en población adulta, siendo escasos los estudios enfocados en población infantil, reportándose mayor mortalidad en comparación a la registrada en niños (30\% vs 1 al 11\%) ${ }^{(6,8,12,13)}$. En nuestra casuística la mortalidad fue elevada (21\%), situación que podría atribuirse a cepas productoras de leucocidina Panton-Valentine, considerando que la forma de presentación más frecuentemente observada fue la neumonía necrotizante. Un estudio reciente realizado en nuestro país, el cual analizólos factores de virulencia en 113 cepas comunitarias de S. aureus, destacó la portación de leucocidina Panton - Valentine como principal factor de virulencia en el $58 \%$ de las muestras analizadas ${ }^{(12)}$.

Hemos encontrado que los factores que se asociaron a mortalidad en nuestra cohorte de niños fueron la presencia de comorbilidad al ingreso, la presencia de choque, saturación de $\mathrm{O}<94$, localización en múltiple focos, trombocitopenia y aislamiento de 
SAMR. Esto coincide con unmetaanálisisreciente sobre bacteriemia por S.aureus que incorporó siete estudios de cohorte en población pediátrica, el cual concluyó que la resistencia a la meticilina fue un factor de riesgo independiente para mortalidad ${ }^{(10)}$. En nuestra casuística la resistencia a meticilina del $S$. aureus aumentó la chance de morir en 2,6 veces $(p=0.03)$ (IC 95\% de 1.1-6.7). Otros grupos de investigadores como Rana Hamdy ${ }^{(13)}$, Isaac ${ }^{(7)}$ y Frederiksen ${ }^{(6)}$ encontraron asociación significativa entre meticilino-resistencia y mortalidad. Estudios prospectivos podrán valorar la participación de otros factores de virulencia en la mortalidad de los niños con bacteremia por $S$. aureus.

\section{REFERENCIAS BIBLIOGRÁFICAS}

1. Larru B, Gong W, Vendetti N, Sullivan KV, Localio R, Zaoutis TE, et al. Bloodstream Infections in Hospitalized Children: Epidemiologyand Antimicrobial Susceptibilities. Ped Infect Dis J 2016; 35:507-510.

2. Jones RN,Guzman-Blanco M, Gales AC, Gallegos B, Castro AL, Martino MD, et al. Susceptibility rates in LatinAmerican nations: report from a regional resistance surveillance program (2011). Braz J Infect Dis 2013; 17:672-81.

3. Suryati BA, Watson M. Staphylococcus aureus bacteraemia in children: a 5-year retrospective review. J PaediatrChildHealth2002;38:290-4.

4. Araya S, Troche A, Amarilla S, Sanabria G, Zarate C, Galeano F, et al. Factores pronósticos de mortalidad en niños con Bacteriemias Adquiridas en la Comunidad (BAC) hospitalizados en un Centro de Referencia del Paraguay. Pediatr. (Asunción). 2018; 45(1):17-24.

5. Kaplan SL, Hulten KG, Gonzalez BE, Hammerman WA, Lamberth L, Versalovic J, et al. Three-yearsurveillance of community-acquiredStaphylococcusaureusinfections in children. Clin Infect Dis 2005; 40:1785-91.

6. Frederiksen MS, Espersen F, Frimodt-Møller N,Jensen AG, Larsen AR, Pallesen LV, et al. Changing epidemiology of pediatric Staphylococcus aureus bacteremia in Denmark from 1971 through 2000. Pediatr Infect Dis J 2007; 26:398.-405.

7. Isaacs D, Fraser S, Hogg G, Li HY. Staphylococcus aureus infections in Australasian neonatal nurseries. Arch Dis Child Fetal Neonatal Ed. 2004;89(4): F331-F335.

\section{CONCLUSIONES}

El presente estudio de Bacteriemia de origen comunitario por $S$. aureus demuestra la elevada frecuencia de $S$. aureus meticilino resistente, así como la marcada severidad en niños, ya que el $40 \%$ de los pacientes requirió ingreso a UCI, el $38 \%$ presentó choque al ingreso y el $21 \%$ falleció.

Se identificaron factores de riesgos asociados a mortalidad tales como: la comorbilidad, la presencia de focos múltiples, choque, saturación de Oxigeno < $94 \%$, trombocitopenia, anemia severa y resistencia a meticilina

8. Paganini H, DellaLattaa P, Sotoa Adriana, Casimir L, Mónaco A, Verdaguer V, et al.Bacteriemias por Staphylococcusaureus adquiridas en la comunidad: 17 años de experiencia en niños de la Argentina.Arch Argent Pediatr2010;108:311-17.

9. Liu VX, Fielding-Singh V, Greene JD, Baker JM, Iwashyna TJ, Bhattacharya J, et al. The Timing of Early Antibiotics and Hospital Mortality in Sepsis. Am J RespirCrit Care Med.2017; 196:856-63.

10. Kumarachandran G, Johnson JK, Shirley D-A. Graffunder E, Heil EL. Predictors of adverse outcomes in c h i l d re n w th Staphylococcus aureusbacteremia.JPediatrPharmacolTher 2017; 22:218-26.

11. Vanderkooi OG, Gregson DB, Kellner JD, Laupland KB. Staphylococcus aureus bloodstream infections in children: A population-based assessment. Paediatr Child Health 2011; 16:276-80.

12. Kobayashi D, Yokota K, Takahashi O, Arioka H, Fukui Tur. A predictive rule for mortality of inpatients with Staphylococcus aureusbacteraemia: A classification and regression tree analysis. European J InternMed. 2014; 25:914-8.

13. Acosta F, Basualdo W, Castro H, Campuzano A, Macchi M, Ortellado de Canese J, et al. Análisis MLVA y perfil de virulencia de aislamientos de Staphylococcusaureus resistentes a la meticilina adquiridos en la comunidad causantes de infecciones pediátricas en Paraguay. Rev Argent Microbiol. 2018;50:151-56. 